\title{
BMJ Open Factors associated with the occurrence of prehospital medical interventions provided by physicians among non- trauma patients: a single-centre retrospective observational study in Japan
}

\author{
Tomohiro Abe, ${ }^{01,2}$ Kenshi Iwatani, ${ }^{1}$ Takeshi Aoyama, ${ }^{1}$ Tatsunori Ameda, ${ }^{1}$ \\ Hidenobu Ochiai ${ }^{2}$
}

To cite: Abe T, Iwatani K, Aoyama T, et al. Factors associated with the occurrence of prehospital medical interventions provided by physicians among non-trauma patients: a single-centre retrospective observational study in Japan. BMJ Open 2019;9:e029186. doi:10.1136/ bmjopen-2019-029186

- Prepublication history and additional material for this paper are available online. To view these files, please visit the journal online (http://dx.doi. org/10.1136/bmjopen-2019029186).

Received 15 January 2019 Revised 03 July 2019 Accepted 25 July 2019

Check for updates

(C) Author(s) (or their employer(s)) 2019. Re-use permitted under CC BY-NC. No commercial re-use. See rights and permissions. Published by BMJ.

${ }^{1}$ Department of Emergency Medicine, Miyazaki Prefectural Miyazaki Hospital, Miyazaki, Japan

${ }^{2}$ Department of Trauma and Critical Care Medicine, University of Miyazaki Hospital, Miyazaki, Japan

Correspondence to Dr Tomohiro Abe; tomohiro_abe@med.miyazakiu.ac.jp

\section{ABSTRACT}

Objectives Physician-staffed prehospital units are widely used in many countries. The criteria for predicting fatal injury are well recognised for trauma victims, but there are no criteria for predicting critical condition for nontrauma patients. This study aimed to identify the factors associated with non-trauma cases receiving prehospital interventions by physicians.

Design Retrospective observational study.

Setting Physician-staffed prehospital unit (car) at a single-base hospital in a suburban city in Japan.

Participants Participants were 1058 non-trauma patients who received prehospital medical examinations from April 2014 to December 2017.

Outcome measures The outcome was the occurrence of physician-only interventions (POIs) exceeding paramedics' competencies. Univariate analysis and multiple logistic regression analysis were performed. Patient's age and gender, presumed disease category, type of location of the emergency, time of alarm, activation time, activator's occupation, time to arrival, transportation time and the destination facility were included as covariates.

Results POls were provided to $380(36 \%)$ patients.

Patient's age, presumed disease category, type of location of the emergency, activator's occupation, time to arrival, transportation time and the destination facility were identified as potential independent factors. Multiple logistic regression analysis found that patient's age, presumed disease category, type of location of the emergency, transportation time and destination facility were the significant independent factors. Transportation times of more than $15 \mathrm{~min}$ (adjusted ORs (AORs) $=4.17$, $95 \% \mathrm{Cl} 2.59$ to $6.72, \mathrm{p}<0.01)$ or 10 to $14 \mathrm{~min}(\mathrm{AOR}=3.66$, $95 \% \mathrm{Cl} 2.32$ to $5.79, \mathrm{p}<0.01)$ and patient age of $40-59$ years $(A 0 R=3.16,95 \% \mathrm{Cl} 1.66$ to $6.01, p<0.01)$ were the strongest independent factors.

Conclusions This study identified the factors associated with non-trauma cases receiving prehospital POls.

Patient's age, presumed disease category, type of location of the emergency and transportation time are independent factors associated with requiring POls.
Strengths and limitations of this study

- This was a first study aiming to identify the factors associated with non-trauma cases receiving prehospital medical interventions by physicians.

- The outcome measure was the occurrence of prehospital physician-only interventions.

- Patient's age, presumed disease category, location of the emergency, longer transportation time and higher level of destination facility were independent factors predicting the occurrence of prehospital medical care by physicians.

- A limitation of the study was that it did not address which patients would benefit from prehospital medical interventions by physicians because patient outcomes, including mortality, were not analysed.

- This was a single-centre retrospective study, so its results may be dependent on regional factors such as the availability of a prehospital physician delivery system and the available time and medical resources.

\section{INTRODUCTION}

Physician-staffed prehospital units, such as helicopter emergency medical services and rapid response vehicles, are currently widely used in many countries, especially in Europe. ${ }^{1}$ These systems have the benefit of delivering medical resources and providing advanced care by physicians during the out-of-hospital phase. In contrast, prehospital systems where advanced care is performed by trained emergency medical technicians and paramedics have been developed in countries such as the USA, Canada, Australia and New Zealand. ${ }^{2}$ Depending on their level of certification, emergency medical technicians and paramedics can perform a variety of prehospital 
medical interventions, such as medication administration and intubation.

In Japan, physician-staffed prehospital units have been developed nationwide. ${ }^{34}$ The Japanese prehospital emergency medical system has incorporated the philosophies of two types of systems: Emergency medical teams generally transport patients to the hospital, and physician-staffed prehospital units are asked to join these emergency medical teams as needed because of the limited competencies of paramedics in Japan.

Early request and dispatch of physician-staffed prehospital units is essential for allowing physicians to reach critically ill patients earlier. For trauma patients, it has been shown that advanced medical care from physician-staffed prehospital emergency services is beneficial. ${ }^{5-8}$ Some criteria for the early recognition of seriously injured victims - mainly based on the mechanisms of injury-have been well described, ${ }^{79}$ but there are few tools available for the early recognition of critically ill patients. There is a trade-off inherent in this situation: lower thresholds for activating physician-staffed prehospital units will result in more overactivation of the units, whereas higher thresholds will result in increased risks of underestimation and delays in providing critical interventions. ${ }^{10}$ Because of the nature of the emergency setting, call centre staff members receive calls reporting a wide variety of symptoms, and most calls indicate that the patient is potentially critically ill. This situation makes it difficult for call centre staff members to judge whether they should activate physician-staffed prehospital units. Establishing criteria for dispatching physician-staffed prehospital units that evaluate which patients are critically ill and in need of earlier intervention is critical, also for non-trauma cases.

We conducted a study aiming to identify the characteristics of non-trauma cases receiving prehospital advanced medical interventions that are beyond the competencies of paramedics and must be performed by physicians in a physician-staffed prehospital unit.

\section{MATERIALS AND METHODS}

\section{Physician-staffed car system}

The physician-staffed prehospital unit examined in this study is a rapid response car system (referred to here as the physician-staffed car system (PSCS)). The base hospital for the PSCS is Miyazaki Prefectural Miyazaki Hospital (MPMH), located in the centre of Miyazaki city, the capital city of Miyazaki prefecture. The PSCS car is staffed by one or two physicians, one nurse and a trained driver. The car operates in and around Miyazaki city, which has a population of approximately 300000 people and an area of approximately $10 \times 5$ kilometres.

The dispatch criteria for the PSCS are the patient needs or seems to need urgent intervention because of a life-threatening condition'. There are no detailed categories or algorithms that dispatchers, who are fire department staff members, can use for the easy and rapid activation of the PSCS. Dispatchers, including call centre staff members, are periodically educated through meetings with medical officers. When these fire department staff members recognise that a case meets or seems to meet the criteria, they activate the PSCS via a direct phone line to the MPMH. The fire department can always activate the system from 08:00 to 17:00. If resources such as manpower are available, the PSCS dispatch also operates outside of this timeframe. The car dispatches from MPMH to the location of the emergency. At the scene, the medical staff members work together with the ambulance team. Physicians provide medical interventions, select a destination facility according to the patient's severity and transport the patient by ambulance, along with ambulance staff members. There is a three-tier system of destination facilities, with facilities classified by their capabilities: several primary care centres/clinics, one general hospital (MPMH) and one tertiary care centre (university hospital).

\section{Study subjects}

This study was a retrospective observational study at a single centre. We surveyed data from the records of non-trauma patients who received prehospital care from the physicians of MPMH's PSCS from April 2014 to December 2017. Exclusion criteria were (1) the request for the PSCS was known to result from external causes (eg, foreign body airway obstruction); (2) the patient was known to be injured at the time of the request for the PSCS; (3) the patient was aged $<16$ years; (4) information about the case suggested that the patients were experiencing anaphylaxis (eg, dyspnoea following a bee sting); (5) patient's information from attending ambulance staff led physicians to decide to dispatch the PSCS car; (6) the PSCS mission was cancelled or aborted or (7) incomplete data were obtained.

We examined data on the patient's age and sex, the patient's symptoms, the type of location of the emergency, the time of alarm, the activation time (the time from the alarm to the request for the PSCS), the activator's occupation (call centre staff at the fire department or ambulance staff), the time to arrival (the time from the PSCS dispatch to the PSCS reaching the patient), the time spent on scene (the time from the PSCS reaching the patient to the start of transportation) and the transportation time (the time from the start of transportation to arrival at the hospital). These variables, except for the time spent on scene, were treated as covariates in the multiple logistic regression analysis. At the time of the study, we categorised the patients' symptoms by presumed organ system disease category as follows: respiratory (eg, dyspnoea), cardiovascular (eg, chest or back pain), consciousness/ neurological (eg, loss of consciousness, paralysis, dysarthria, seizure), cardiopulmonary arrest (CPA) (eg, coma and not breathing) and the other (eg, precipitate labour). The location of the emergency was categorised as follows: home, welfare facility, public place (eg, a store, bank, town office), patient's workplace/school or street. Decisions about subject inclusion/exclusion criteria and 
classification by presumed disease category were made through discussion among the authors.

\section{Outcome}

The outcome was physician-only intervention (POI) being performed before arrival at the hospital. POIs included all interventions exceeding the competencies of paramedics in Japan but did not include any diagnostic examinations (eg, ultrasound, blood glucose testing, ECG). The competencies of paramedics in Japan are as follows: advanced airway management (including tracheal intubation), intravenous cannulation and administration of epinephrine for patients with CPA; glucose administration for patients with hypoglycaemia and peripheral intravenous cannulation and fluid administration for patients with shock. All of these interventions require telemedical assistance by physicians.

\section{Statistical analysis}

The continuous variables were coded categorically. The ordinal variables were coded as follows: age was categorised as (1) younger than 20 years, (2) 20-39 years, (3) 40-59 years, (4) 60-79 years or (5) 80 years or older; the time of alarm was categorised as (1) before 10:00, (2) 10:00-12:00, (3) 12:00-14:00, (4) 14:00-16:00 or (5) after 16:00; other time variables were divided into (1) less than 5 min, (2) 5-9 min, (3) 10-14 min or (4) 15 min or more. We used $\chi^{2}$ tests for all categorical variables. Fisher's exact tests were used instead of $\chi^{2}$ tests when more than $20 \%$ of the cells had expected values less than 5 , according to Cochran's rule.

We used logistic regression analysis to identify the independent variables predicting the occurrence of POIs. We included all variables simultaneously and identified significant independent variables. We assessed multicollinearity among the variables using the following criteria: phi coefficient over 0.7 when both variables were binary; Cramér's $\mathrm{V}$ over 0.7 when one or both variables had three or more values and Spearman's rank correlation coefficient over 0.7 when both variables were ordinal. We created a compiled group with groups without differences of ORs for POIs between the groups for each variable's reference group to assess the independent effects of the examined variables. We built the final multiple logistic regression model estimating the occurrence of POIs and evaluated the model with all of the explanatory variables and the final model using the area under the receiver operating characteristic curve (ROC-AUC). We used the HosmerLemeshow test to assess the goodness of fit of the logistic regression equation. We assessed outlying cases more than three SDs from the mean. Statistical significance was defined as alpha less than $5 \%$ for all statistics. We used SPSS software V.23 (IBM SPSS Statistics, IBM Corp) for all of the statistical analyses.

\section{Patient and public involvement}

None of the patients or the public were involved in the design or analysis of this study. We presented the

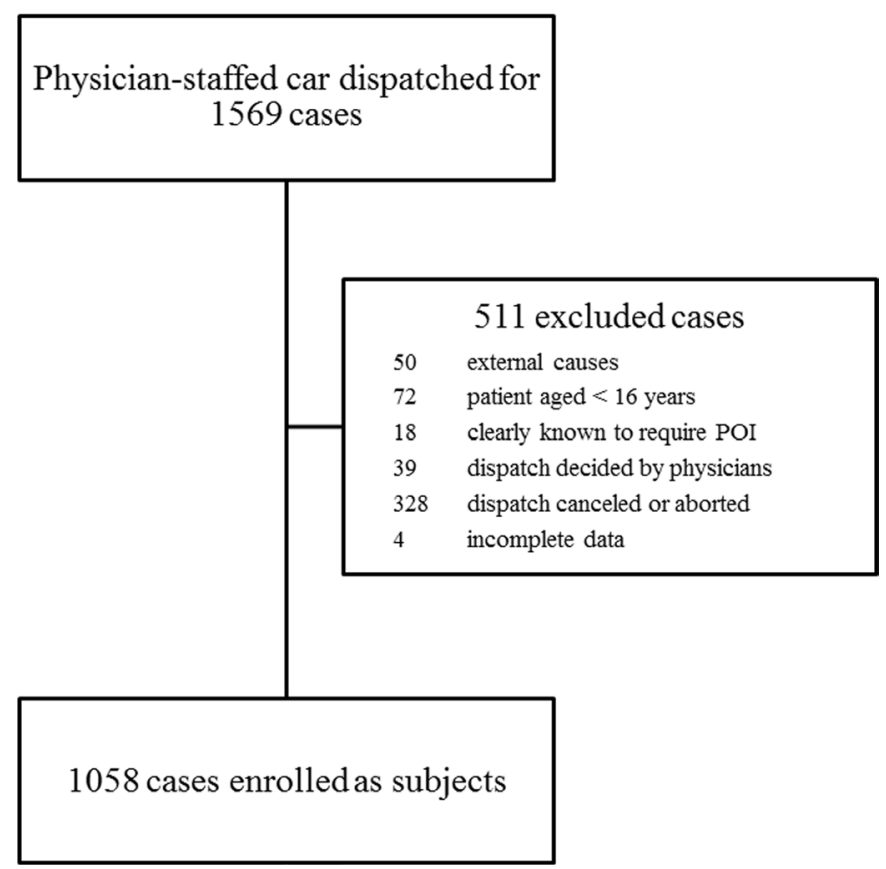

Figure 1 Enrolment of the subjects. POI, physician-only intervention.

preliminary results of the study at a regional meeting on emergency medicine. We will submit a summary of the results to a hospital magazine for the public to inform to the general population of the results after the publication of this article.

\section{RESULTS}

\section{Study subjects}

The physician-staffed car was dispatched for 1569 non-trauma cases during the study period. We excluded 511 patients, leaving 1058 patients who were eligible study subjects (figure 1). The patients' median (25th-75th percentile) age was 74 years (60-84 years), and 612 (58\%) patients were men. The most frequent presumed disease category was consciousness disorder $(\mathrm{n}=580,55 \%)$, and the most common type of location of the emergency was a home $(\mathrm{n}=573,54 \%)$. The activator of the PSCS was usually a call centre staff member $(n=907,86 \%)$. The activation time was less than $5 \mathrm{~min}$ in $81 \%$ of the examined cases. The medians (25th-75th percentiles) for the time parameters were as follows: 2 (1-4) min for activation time, 7 (4-11) min for time to arrival, 9 (6-12) min for time spent on scene and $8(5-12)$ minutes transportation time. A total of $785(74 \%)$ patients were transported to the MPMH; only $3.5 \%$ of the patients were transported to the tertiary care centre (table 1 ).

A total of $380(36 \%)$ patients received POIs before arrival at the hospital. The most frequent POI was the administration of medication $(\mathrm{n}=354,33 \%)$, followed by endotracheal intubation $(\mathrm{n}=38,4 \%)$ and catheter insertion through two or more intravenous routes $(n=23,2 \%$; table 2). 
Table 1 Demographic characteristics of the study subjects

Total $(\mathrm{N}=1058)$

\begin{tabular}{|cc}
\hline Age (years) & $20(2)$ \\
$<20$ & $59(6)$ \\
$20-39$ & $172(16)$ \\
$40-59$ & $406(38)$ \\
$60-79$ & $401(38)$ \\
$\geq 80$ & \\
\hline Sex & $612(58)$ \\
\hline Male & $446(42)$ \\
\hline Female & $74(7)$ \\
\hline Presumed disease category & $190(18)$ \\
\hline Respiratory & $580(55)$ \\
\hline Cardiovascular & $208(20)$ \\
\hline Consciousness/neurological & $6(1)$ \\
\hline CPA & \\
\hline Other & $573(54)$ \\
\hline Location of the emergency & $125(12)$ \\
\hline Home & $205(19)$ \\
\hline Welfare facility & $56(9)$ \\
\hline Public place & \\
\hline Workplace/school & \\
\hline Street & \\
\hline
\end{tabular}

Time of alarm

\begin{tabular}{|c|c|}
\hline Before 10:00 & $277(26)$ \\
\hline $10: 00-12: 00$ & $249(24)$ \\
\hline $12: 00-14: 00$ & $185(17)$ \\
\hline $14: 00-16: 00$ & $225(21)$ \\
\hline After 16:00 & $122(12)$ \\
\hline \multicolumn{2}{|l|}{ Activation time (min) } \\
\hline$<5$ & $857(81)$ \\
\hline $5-9$ & $114(11)$ \\
\hline $10-14$ & $47(4)$ \\
\hline$\geq 15$ & $40(4)$ \\
\hline \multicolumn{2}{|l|}{ Activator's occupation } \\
\hline Call centre staff member & $907(86)$ \\
\hline Ambulance staff member & $151(14)$ \\
\hline \multicolumn{2}{|l|}{ Time to arrival (min) } \\
\hline$<5$ & $321(30)$ \\
\hline $5-9$ & $419(40)$ \\
\hline $10-14$ & 204 (19) \\
\hline$\geq 15$ & $114(11)$ \\
\hline \multicolumn{2}{|l|}{ On scene time (min) } \\
\hline$<5$ & $115(11)$ \\
\hline $5-9$ & $518(49)$ \\
\hline $10-14$ & $297(28)$ \\
\hline$\geq 15$ & $128(12)$ \\
\hline
\end{tabular}

Continued
Table 1 Continued

Total $(\mathrm{N}=1058)$

Transportation time (min)

\begin{tabular}{lc}
$<5$ & $198(19)$ \\
$5-9$ & $430(41)$ \\
$10-14$ & $239(23)$ \\
$\geq 15$ & $191(18)$ \\
Destination facility & \\
Primary care centre/clinic & $236(22)$ \\
General hospital (MPMH) & $785(74)$ \\
Tertiary care centre & $37(3)$ \\
\hline
\end{tabular}

Values are expressed as $n(\%)$ unless indicated otherwise. CPA, cardiopulmonary arrest; MPMH, Miyazaki Prefectural Miyazaki Hospital.

\section{Univariate analysis}

Patient's age, presumed disease category, type of location of the emergency, activation time, activator's occupation, time to arrival, transportation time and destination facility were found to be potential factors predicting the occurrence of POIs. Patient gender and the time of alarm were not significantly associated with the occurrence of POIs.

More specifically, relatively strong potential factors positively associated with the occurrence of POIs were presumed cardiovascular disease category (unadjusted OR $(\mathrm{UOR})=1.89,95 \%$ CI 1.38 to $2.60, \mathrm{p}<0.01)$, workplace or school as the type of location of the emergency (UOR $=1.78,95 \%$ CI 1.17 to $2.69, \mathrm{p}<0.01)$ and transportation time over $15 \mathrm{~min}$ (UOR=1.77, 95\% CI 1.29 to 2.44 , $\mathrm{p}<0.01$ ). Strong potential factors negatively associated with the occurrence of POIs were transportation time under $5 \mathrm{~min}$ (UOR $=0.39,95 \%$ CI 0.27 to $0.56, \mathrm{p}<0.01$ ), the patient being aged 20-39 years (UOR $=0.44,95 \%$ CI 0.23 to $0.84, \mathrm{p}=0.01$ ) and presumed CPA disease category (UOR $=0.47,95 \%$ CI 0.33 to $0.67, \mathrm{p}<0.01$; see online supplementary file 1 ).

\section{Multiple logistic regression analysis}

Patient's age, presumed disease category, type of location of the emergency and transportation time were found to be independent factors predicting the occurrence of

Table 2 Physician-only interventions provided

\begin{tabular}{lc}
\hline & No (\%) \\
\hline Administration of medication* & $354(33)$ \\
\hline Tracheal intubation* & $38(4)$ \\
Transcutaneous pacing & $0(0)$ \\
Tube thoracostomy & $1(0.1)$ \\
Two or more intravenous catheter insertions & $23(2)$ \\
Pericardiocentesis & $6(1)$ \\
\hline
\end{tabular}

*Epinephrine administration and tracheal intubation for cardiopulmonary arrest patients are not included here. 
OR $(95 \% \mathrm{CI})$

Transportation Time (vs $<5 \mathrm{~min}$.)

$$
\begin{aligned}
& \text { 5-9 min. } \\
& 10-14 \mathrm{~min} . \\
& \geq 15 \mathrm{~min} .
\end{aligned}
$$

Location of the emergency (vs Public place \& Welfare facility)

Home

Street

Workplace/school

Age (vs $<39$ aged)

$$
\begin{aligned}
& 40-59 \\
& 60-79 \\
& \geq 80
\end{aligned}
$$

Presumed disease category (vs CPA \& Others)

$$
\begin{aligned}
& \text { Respiratory } \\
& \text { Cardiovascular } \\
& \text { Consciousness / neurological }
\end{aligned}
$$

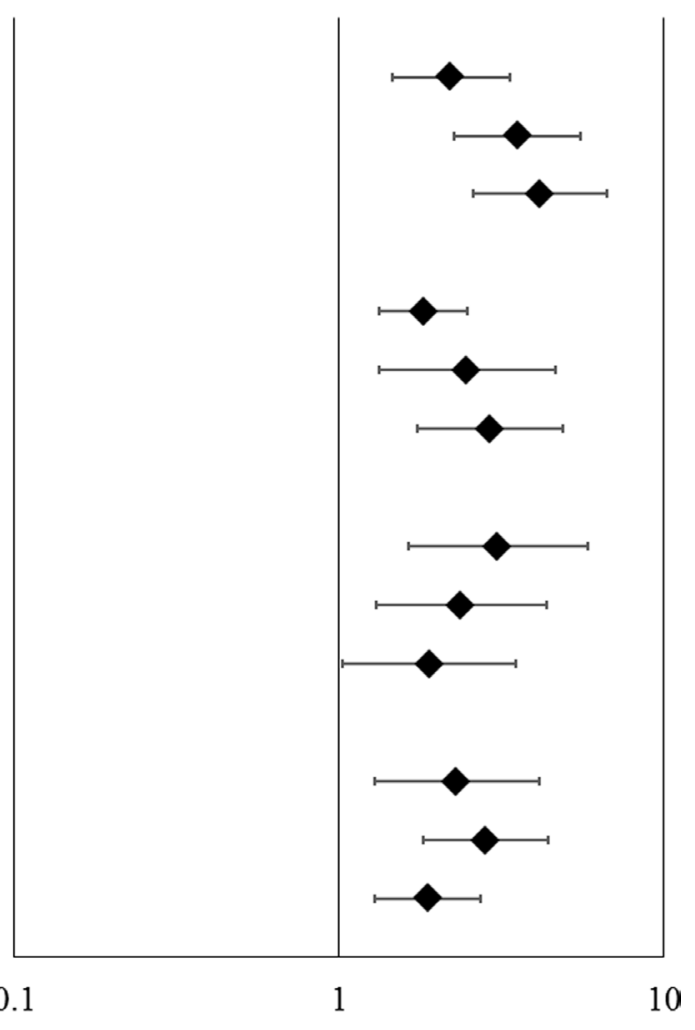

\section{Odds ratios}

Figure 2 Adjusted ORs calculated by multiple logistic regression. CPA, cardiopulmonary arrest; MPMH, Miyazaki Prefectural Miyazaki Hospital.

POIs. Patient's gender, activation time, activator's occupation and the time to arrival were not independent factors predicting the occurrence of POIs. None of the independent factors showed multicollinearity, which would have been indicated by values exceeding 0.7 for the phi coefficient, Cramér's V or Spearman's rank correlation coefficient.

Among the independent factors, the strongest factors that were positively associated with the occurrence of POIs were transportation time over $15 \mathrm{~min}$ (compared with transportation time $<5 \mathrm{~min}$; adjusted $\mathrm{OR}(\mathrm{AOR})=4.14$, $95 \%$ CI 2.58 to $6.67, \mathrm{p}<0.01)$, transportation time from 10 to $14 \mathrm{~min}$ (AOR=3.55, 95\% CI 2.26 to $5.57, \mathrm{p}<0.01$ ), the patient being aged 40-59 years (compared with $<39$ years; $\mathrm{AOR}=3.10,95 \%$ CI 1.63 to $5.87, \mathrm{p}<0.01$ ), presumed cardiovascular disease category (compared with CPA and other categories; AOR=2.83, 95\% CI 1.81 to 4.43 , $\mathrm{p}<0.01$ ) and workplace/school as the type of location of the emergency (compared with welfare facility/public place; $\mathrm{AOR}=2.91,95 \%$ CI 1.74 to $4.89, \mathrm{p}<0.01$; figure 2$)$. The ROC-AUC was 0.70 (95\% CI 0.67 to $0.73, \mathrm{p}<0.01$ ) for the model with all explanatory variables and 0.69 (95\% CI 0.66 to $0.72, p<0.01$ ) for the final model. The $p$ value for the Hosmer-Lemeshow test of the final model was 0.53. Outliers exceeding three SDs were not detected.

\section{DISCUSSION}

We present the factors associated with non-trauma cases requiring prehospital medical treatment provided by physicians. Symptoms associated with the cardiovascular and respiratory systems, the patient being of middle age and prolonged transportation time were found to be independent factors predicting the occurrence of POIs.

Our findings on the factors of presumed cardiovascular and respiratory disease categories were similar to those in previous reports, although a direct comparison was not possible because the outcome measures were different. Christenszen et al reported that a prehospital unit staffed by physicians (anaesthesiologists) reduced mortality only among patients with acute myocardial infarctions or respiratory diseases. ${ }^{11}$ The finding that the middle-age group had the highest odds of receiving POIs seems strange because the proportions of cardiovascular and respiratory diseases, including acute coronary syndrome and chronic obstructive pulmonary disease, are higher among older patients. Considered together with the finding that those with workplace/school as the location of the emergency had the highest odds of receiving POIs, it is possible that physicians may take the patient's background into account when deciding whether to perform medical interventions before reaching the hospital. Patients of middle age who 
are exhibiting cardiovascular or respiratory symptoms are potential cases where POI can be used and may improve the outcome.

The ORs of neurological/consciousness disorder was lower than those of cardiovascular and respiratory disorders. According to a previous study that compared patients with altered mental status receiving prehospital advanced life support and those receiving only basic life support, receiving advanced life support only served to reduce the time spent in the emergency room. ${ }^{12}$ To our knowledge, only prehospital diazepam administration for status epilepticus in children has been reported as effective for reducing the duration of seizures and recurrence likelihood. ${ }^{13}$ In the case of neurological or consciousness symptoms, obtaining information on epileptic status may be the key to deciding whether to request the PSCS.

The CPA group was found to have a lower odds of POIs, compared with other disease categories. This may be explained by the study design because the outcome excluded interventions within paramedics' competencies. Among patients with CPA in this study, the provision of interventions by physicians that were within the competencies of paramedics was $46 \%$ (data not shown). For non-trauma patients with CPA, some studies have shown that physician-provided prehospital interventions had positive outcomes, including an increase in the likelihood of the return of spontaneous circulation and a reduction in mortality. ${ }^{14-16}$ Böttiger et $a l^{15}$ mentioned a possible explanation when citing other reports, arguing that paramedics have difficulties obtaining and maintaining lifesaving skills and that physicians provide higher quality resuscitations. ${ }^{16}$ The effectiveness of physician-staffed prehospital units may be achieved through having physicians reach patients earlier and through physicians directing the care during the all phases, from prehospital to in-hospital. ${ }^{17}$

Prolonged transportation time was found to be an independent factor associated with the occurrence of POIs. This finding may have resulted from physicians' decisions prioritising patient transport over prehospital medical interventions. Factors related to physicians reaching patients earlier, including activation time and time to arrival, were not found to be independently associated with the occurrence of POIs. These findings may suggest that the dispatch criteria should be based on whether a patient needs prehospital medical interventions during transportation rather than on whether a patient needs a physician to reach them earlier. Prehospital systems focusing intently on delivering medical resources earlier might have some adverse effects, including misjudgments resulting in physicians being dispatched to less severe patients when there are multiple alarms, pressure on drivers to reach the scene earlier potentially increasing the risk of automobile accidents ${ }^{18}$ and cost problems.

Physician-staffed prehospital units cannot be available at all times and in all places. ${ }^{3} 19$ In this study, because the existing PSCS dispatch criteria avoided providing detailed descriptions, with the aim of facilitating easy and rapid activation of the PSCS, we were able to identify the characteristics of cases where POIs occurred across a broad range of included subjects. The POI rate was 36\% after excluding cancelled dispatches, revealing that the dispatch criteria allowed significant overactivation of the PSCS. Our results suggest that obtaining more accurate information from the caller is important and that analysis by call centre staff members may improve the balance between accurate activation and ensuring that physicians reach patients in a timely fashion. The impact of other factors, such as the patient's vital signs, on the occurrence of POIs might be stronger than the impact of the variables included in this study because the ROC-AUCs were similar for the model including all explanatory variables and the final model.

\section{Study limitations}

This study was designed with the aim of identifying the factors predicting the occurrence of prehospital POIs. The ROC-AUC for the model with all explanatory variables was relatively low $(0.70)$, which may suggest that other factors not included in this study are stronger factors for POI occurrence. The main limitation of this study was that it did not consider final diagnoses, treatments or prognostic parameters, including mortality after admission to the hospital. We therefore cannot make any conclusions about the characteristics of cases that benefit from physician-staffed prehospital interventions. The results of this study may be useful for establishing PSCS dispatch criteria, but they should not be used for making inferences about the effectiveness of the PSCS. Furthermore, studies examining prehospital care depend on the particularities of the regional setting, such as geographic factors, available resources and legal and political issues. Additionally, our study covered only daytime hours, which may have introduced selection bias in that patient groups with conditions linked to circadian rhythms ${ }^{20-22}$ were not equally likely to be included in the study.

\section{CONCLUSION}

Our study identified characteristics of non-trauma cases associated with receiving prehospital medical interventions by the PSCS. Cardiovascular and respiratory symptoms, long transportation times, patients' being middle aged and the workplace/school as the location of the emergency were found to be independent factors predicting the occurrence of POIs. These independent factors may indicate that the potential role of staffing prehospital response units with physicians depends not on early intervention but on the safe transportation of critically ill patients.

Acknowledgements We would like to thank Hiroaki Hidaka and Toshinobu Kushima for their cooperation in this study. We also thank Jennifer Barrett, PhD, from Edanz Group (www.edanzediting.com/ac) for editing a draft of this manuscript.

Contributors TAb designed the study, performed the statistical analysis, wrote the manuscript and submitted the article. KI and TAo collected the relevant references. 
TAm and HO supervised the development of the article. All authors approved the final manuscript.

Funding The authors have not declared a specific grant for this research from any funding agency in the public, commercial or not-for-profit sectors.

Competing interests None declared.

Patient consent for publication Not required.

Ethics approval The institutional review board at Miyazaki Prefectural Miyazaki Hospital approved this study protocol (18-14).

Provenance and peer review Not commissioned; externally peer reviewed.

Data availability statement № additional data are available.

Open access This is an open access article distributed in accordance with the Creative Commons Attribution Non Commercial (CC BY-NC 4.0) license, which permits others to distribute, remix, adapt, build upon this work non-commercially, and license their derivative works on different terms, provided the original work is properly cited, appropriate credit is given, any changes made indicated, and the use is non-commercial. See: http://creativecommons.org/licenses/by-nc/4.0/.

\section{REFERENCES}

1. Al-Shaqsi S. Models of international emergency medical service (EMS) systems. Oman Med J 2010;25:320-3.

2. Shah MN. The formation of the emergency medical services system. Am J Public Health 2006;96:414-23.

3. Igarashi $\mathrm{Y}$, Yokobori $\mathrm{S}$, Yamana $\mathrm{H}$, et al. Overview of doctor-staffed ambulance use in Japan: a nationwide survey and 1-week study. Acute Med Surg 2018;5:316-20.

4. Nishikawa W, Yamano Y. An overview of the development of helicopter emergency medical services in Japan. Air Med $J$ 2010;29:288-91.

5. Garner A, Rashford S, Lee A, et al. Addition of physicians to PARAMEDIC helicopter services decreases blunt trauma mortality. ANZ J Surg 1999;69:697-701.

6. Wilson SL, Gangathimmaiah V. Does prehospital management by doctors affect outcome in major trauma? A systematic review. $J$ Trauma Acute Care Surg 2017;83:965-74.

7. Den Hartog D, Romeo J, Ringburg AN, et al. Survival benefit of physician-staffed helicopter emergency medical services (HEMS) assistance for severely injured patients. Injury 2015;46:1281-6.

8. Blom MC, Aspelin L, Ivarsson K. Propensity for performing interventions in pre-hospital trauma management - a comparison between physicians and non-physicians. $J$ Trauma Manag Outcomes 2014;8.

9. Wigman LD, van Lieshout EMM, de Ronde G, et al. Trauma-Related dispatch criteria for helicopter emergency medical services in Europe. Injury 2011;42:525-33.

10. Parikh PP, Parikh P, Guthrie B, et al. Impact of triage guidelines on prehospital triage: comparison of guidelines with a statistical model. J Surg Res 2017;220:255-60.

11. Christenszen EF, Melchiorsen H, Kilsmark J, et al. Anesthesiologists in prehospital care make a difference to certain groups of patients. Acta Anaesthesiol Scand 2003;47:146-52.

12. Adams J, Aldag G, Wolford R. Does the level of prehospital care influence the outcome of patients with altered levels of consciousness? Prehosp Disaster Med 1996;11:101-4.

13. Alldredge BK, Pharm D, Wall DB, et al. Effect of prehospital treatment on the outcome of status epilepticus in children. Pediatr Neurol 1995;12:213-6.

14. Hamilton A, Steinmetz J, Wissenberg M, et al. Association between prehospital physician involvement and survival after out-ofhospital cardiac arrest: a Danish nationwide observational study. Resuscitation 2016;108:95-101.

15. Böttiger BW, Bernhard M, Knapp J, et al. Influence of EMSphysician presence on survival after out-of-hospital cardiopulmonary resuscitation: systematic review and meta-analysis. Crit Care 2015;20.

16. Olasveengen TM, Lund-Kordahl I, Steen PA, et al. Out-of hospital advanced life support with or without a physician: effects on quality of CPR and outcome. Resuscitation 2009;80:1248-52.

17. von Vopelius-Feldt J, Benger JR. Should physicians attend out-ofhospital cardiac arrests? Resuscitation 2016;108:A6-7.

18. Eksi A, Celikli S, Catak I. Effects of the institutional structure and legislative framework on ambulance accidents in developing emergency medical services systems. Turkish J Emerg Med 2015;15:126-30.

19. Hyde P, Mackenzie R, Ng G, et al. Availability and utilisation of physician-based pre-hospital critical care support to the NHS ambulance service in England, Wales and Northern Ireland. Emerg Med J 2012;29:177-81.

20. Thompson DR, Sutton TW, Jowett NI, et al. Circadian variation in the frequency of onset of chest pain in acute myocardial infarction. $\mathrm{Br}$ Heart J 1991;65:177-8.

21. Manfredini R, Portaluppi F, Zamboni $P$, et al. Circadian variation in spontaneous rupture of abdominal aorta. Lancet 1999;353:643-4

22. Hetzel MR, Clark TJ, Branthwaite MA. Asthma: analysis of sudden deaths and ventilatory arrests in hospital. Br Med J 1977;1:808-11. 